\title{
Brain Activation Associated with Attentional Bias in Smokers is Modulated by a Dopamine Antagonist
}

\author{
Maartje Luijten*, ${ }^{*, 2}$, Dick J Veltman ${ }^{3,4}$, Robert Hester ${ }^{5}$, Marion Smits ${ }^{2}$, Lolke Pepplinkhuizen ${ }^{6}$ and \\ Ingmar HA Franken ${ }^{1,7}$ \\ IInstitute of Psychology, Erasmus University Rotterdam, Rotterdam, The Netherlands; ${ }^{2}$ Department of Radiology, Erasmus MC — University \\ Medical Center Rotterdam, Rotterdam, The Netherlands; ${ }^{3}$ Department of Psychiatry, Academic Medical Center, University of Amsterdam, \\ Amsterdam, The Netherlands; ${ }^{4}$ Department of Psychiatry, VU University Medical Center, Amsterdam, The Netherlands; ${ }^{5}$ Department of \\ Psychological Sciences, University of Melbourne, Melbourne, Victoria, Australia; ${ }^{6}$ Psychiatric Centre Bavo-Europoort, Capelle ald IJssel, The \\ Netherlands; ' Department of Child and Adolescent Psychiatry, Erasmus MC — Sophia Children's Hospital, University Medical Center Rotterdam, \\ Rotterdam, The Netherlands
}

\begin{abstract}
Attentional bias in substance-dependent individuals is the tendency to automatically direct the attention to substance-related cues in the environment. Attentional bias is known to be associated with clinical measures such as relapse or successful quitting in smokers. It has been suggested that attentional bias emerges as a consequence of dopaminergic activity evoked by substance-related cues. The current functional magnetic resonance imaging study employed a dopaminergic challenge in order to test whether brain activation associated with attentional bias in smokers could be modulated by a dopamine antagonist. A total of 25 smokers were compared with 24 controls. Participants were scanned twice while performing a pictorial attentional bias task. Haloperidol (2 mg), a selective D2/D3 dopamine antagonist, or placebo was orally administered $4 \mathrm{~h}$ before each scanning session in a double-blind randomized cross-over design. Imaging analyses were performed in a priori selected regions of interest. Results showed that smokers had enhanced brain activation compared with controls in the dorsal anterior cingulate cortex (dACC), right dorsolateral prefrontal cortex ( $r$-DLPFC), and left superior parietal lobe (I-SPL) after placebo. Group $\times$ medication interactions were found in the dACC and r-DLPFC, with no differences between groups in these regions after haloperidol. The current findings suggest that a pharmacologically induced reduction in dopamine normalizes brain activation associated with attentional bias in the dACC and DLPFC in smokers, probably because salience of these cues is no longer detected when dopamine activity is reduced.

Neuropsychopharmacology (2012) 37, 2772-2779; doi:10.1038/npp.2012.143; published online I August 2012
\end{abstract}

Keywords: attentional bias; dopamine; smokers; functional magnetic resonance imaging

\section{INTRODUCTION}

Substance abuse and addiction are associated with enhanced processing of substance-related cues (eg, Franken et al, 2003; Kuhn and Gallinat, 2011). Attentional bias is one of the mechanisms underlying enhanced processing of these cues and is defined as the tendency of substance-dependent people to automatically and involuntarily allocate and maintain their attention to conditioned drug cues (for reviews, see Field and Cox, 2008; Franken, 2003; Robbins and Ehrman, 2004). Attentional bias has been linked to craving (Field et al, 2009b) as well as to the temptation to use substances (Waters et al, 2012), treatment outcome (Carpenter et al, 2006; Cox et al, 2002), and relapse rates

*Correspondence: M Luijten, Institute of Psychology, Erasmus University Rotterdam, PO Box 1738, Rotterdam, 3000 DR, The Netherlands, Tel: + 31 104089729, Fax: + 31 104089009, E-mail: luijten@fsw.eur.nl

Received I6 May 2012; revised 25 June 2012; accepted 3 July 2012
(Marissen et al, 2006; Waters et al, 2003). Preliminary evidence has further suggested that attentional bias extinction training reduces conditioned cigarette craving in smoking males (Attwood et al, 2008) and drinking behavior in alcohol-dependent patients (Fadardi and Cox, 2009; Schoenmakers et al, 2010). However, it seems that a single training session is not successful to reduce smoking behavior (Attwood et al, 2008; Field et al, 2009a; McHugh et al, 2010).

Theoretical models propose that attentional bias is a consequence of a dopamine signal that triggers attention to substance-related cues (Franken, 2003; Robinson and Berridge, 2003). After repeated drug intake, substancerelated cues become conditioned cues and elicit dopaminergic activity (Volkow et al, 2006; Wong et al, 2006; Zijlstra et al, 2008) thereby signaling the expectation of a future reward (ie, the intake of the substance of abuse). Gradually, the dopaminergic system becomes sensitized for substancerelated cues so that they become extremely salient, become the focus of attention and elicit behaviors like drug seeking 
and consumption (Phillips et al, 2003; Robinson and Berridge, 2008). Consequently, it can be predicted that attentional bias will be attenuated when dopamine is no longer able to signal the salience of conditioned substancerelated cues. A few studies tested this hypothesis. Two studies used acute tyrosine/phenylalanine depletion to reduce dopamine levels in smokers (Hitsman et al, 2008; Munafo et al, 2007). Both studies found that attentional bias in smokers was reduced when dopamine levels were decreased. Similar results were found in heroin users, in which attentional bias was attenuated after a single dose of the dopamine antagonist haloperidol (Franken et al, 2004). As these studies did not measure brain activation, it is not yet known whether a pharmacologically induced reduction in dopamine also reduces brain activation in those regions involved in salience detection and attentional bias. Only recently progress has been made to elucidate the neurobiological substrate of attentional bias. Functional magnetic resonance imaging (fMRI) has shown that attentional bias is associated with increased activation in brain regions innervated by dopaminergic projection such as the ACC and the ventral striatum (Janes et al, 2010a; Luijten et al, 2011; Nestor et al, 2011; Vollstadt-Klein et al, 2012). In addition, dorsolateral and inferior frontal regions, as well as the insula, amygdala, superior parietal, and superior and middle temporal gyri were implicated in attentional bias for substance-related cues (Ersche et al, 2010; Hester and Garavan, 2009; Janes et al, 2010a; Luijten et al, 2011; Vollstadt-Klein et al, 2012). Ersche et al (2010) showed that a dopamine agonist enhanced attentional bias and associated brain activation in the left ventral prefrontal cortex and the cerebellum in high-compulsive stimulant dependent individuals, whereas it reduced activation in these regions in lowcompulsive stimulant users. The Ersche et al study could not demonstrate a reduction in attentional bias and associated brain activation following administration of the dopamine antagonist amisulpride. Consequently, it is still unknown whether a pharmacologically induced reduction in dopamine levels could normalize brain activation associated with attentional bias. In this study, attentional bias-related brain activation was measured twice in smokers and non-smokers using an attentional bias task involving pictorial stimuli (Luijten et al, 2011). The D2/D3 dopamine antagonist haloperidol was used to reduce dopamine levels and was compared with placebo in a double-blind randomized crossover design. Based on theoretical accounts and our previous study in smokers (Luijten et al, 2011), we hypothesized that brain activation associated with attentional bias in dopaminergic innervated regions such as the ACC and other prefrontal regions will normalize in smokers after haloperidol administration. That is, no differences between smokers and non-smokers in attentional bias-related brain activation were expected after haloperidol.

\section{MATERIALS AND METHODS}

\section{Participants}

A total of 25 smokers and 25 non-smokers participated in this study. Data from one non-smoker were discarded because of technical problems during data analyses. The final sample consisted of 25 smokers (mean age $=22.56$ years, $\mathrm{SD}=2.84,18$ males) and 24 non-smokers (mean age $=21.75$ years, $\mathrm{SD}=1.78,14$ males $).$ Smokers smoked at least 15 cigarettes per day (mean $=19.12, \mathrm{SD}=3.37$; range 15-25) for a duration of at least three years (mean $=7.20$, $\mathrm{SD}=3.01$, range $3-14$ ). The average score on the Fagerström Test for nicotine dependence (Vink et al, 2005) for smokers was $3.80, \mathrm{SD}=3.37$, range $1-8$. Non-smokers had smoked ten cigarettes or less during their lifetime (mean $=1.73, \mathrm{SD}=2.62$, range $0-10)$. Participants underwent a medical examination by a psychiatrist to assure eligibility for a single dose of $2 \mathrm{mg}$ oral haloperidol (for details, see Supplementary materials). Exclusion criteria for both groups were (a) current substance abuse or dependence (other than nicotine for smokers), (b) any physical or psychological illness, (c) any use of psychotropic medication or medication that may affect blood circulation and/ or respiration, (d) fMRI contraindications, and (e) left handedness. There were no significant differences between the groups in age, $t(47)=2.40 ; \mathrm{NS}$, or gender, $\chi \quad(1$, $n=49)=0.32$; NS. The study was conducted in accordance with the Declaration of Helsinki and all procedures were carried out after participants signed informed consent. The ethics committee of Erasmus MC-University Medical Centre Rotterdam approved this study.

\section{Dopaminergic Manipulation}

Participants were administered a single oral dose of $2 \mathrm{mg}$ haloperidol and a placebo employing a double-blind randomized cross-over design. Haloperidol is a selective post-synaptic dopamine D2/D3 receptor antagonist. Using positron emission tomography (PET), it has been shown that striatal D2 receptor occupancy $3 \mathrm{~h}$ after administration of $2 \mathrm{mg}$ haloperidol is $18 \%$ and $52 \%$ after $6 \mathrm{~h}$ (Nordstrom et al, 1992). The present fMRI session took place $4 \mathrm{~h}$ after administration which, according to the Nordstrom study, results in about $30 \% \mathrm{D} 2$ receptor occupancy. The dose and time interval was further based on previous studies using haloperidol (Franken et al, 2004, 2008; Mahler and de Wit, 2005) that showed attenuated cue-reactivity in smokers (Mahler and de Wit, 2005) and attentional bias in heroin users (Franken et al, 2004) after haloperidol. No side effects were reported by the participants. Participants' guesses on the type of medication they received for each scanning session were not above chance $(48.97 \%$ of the participants correctly indicated in which test occasion they received haloperidol, $p=0.56$ ).

\section{Procedures}

Two scanning sessions were scheduled that were separated by 1 week. Smokers were not allowed to smoke after taking the medication until scanning was finished to ensure that indirect nicotine effects on dopamine levels did not interfere with the binding of haloperidol to D2/D3 receptors in the brain. Breath carbon monoxide (CO) concentration was measured in all subjects using a calibrated Micro + Smokerlyzer (Bedfont Scientific, Rochester, UK). Smokers completed the FTND (Heatherton et al, 1991; Vink et al, 2005) to measure nicotine dependence on the first scanning session only and the Questionnaire of Smoking Urges (QSU; Cox et al, 2002) to indicate their current subjective craving 
for a cigarette during both scanning sessions. Participants performed the attentional bias line-counting (ABLC) task during fMRI scanning. Smokers indicated their craving levels on a 100-point visual analog scale (VAS) immediately before and after task performance.

\section{Task Paradigm}

The ABLC task was used to measure brain activation related to attentional bias, and has previously been described by Luijten et al (2011). In each trial, a picture with either smoking-related stimuli (people engaged in smoking behavior or smoking-related objects) or neutral stimuli (people engaged in non-smoking behavior or neutral objects) was presented for $900 \mathrm{~ms}$ (Figure 1). Two to five lines were displayed within each picture, with semirandomly distributed spaces between these lines. Instructions for participants varied over blocks. In one block (counting lines), participants were asked to count the number of lines presented in the picture and to press the corresponding button as fast as possible. In the other block (naming pictures), participants had to indicate whether the content of the picture included smoking-related stimuli or neutral stimuli by pressing the corresponding button. Within each block, smoking-related and neutral pictures were semi-randomly presented. Seventy-two trials were presented for each condition: line-counting smoke picture (LCSP), line-counting neutral picture (LCNP), picturenaming smoke picture (PNSP), and picture-naming neutral picture (PNNP). Based on these conditions, four contrasts can be defined for analyses. The main contrast reflecting brain activation associated with attentional bias is the LCSP minus LCNP contrast. For the line-counting condition, smoking cues are unrelated to task performance so that brain activation for LCSP relative to LCNP shows the disruption of ongoing behavior (line counting) because of the enhanced attentional and motivational properties of the smoking pictures. The other three contrasts do not reflect attentional bias and accordingly are not associated with the main focus of this paper. See Supplementary materials for the definition and results of these contrasts.

\section{Data Analyses Questionnaires and Behavioral Performance}

A group (smokers $v s$ non-smokers) $\times$ medication (placebo $v s$ haloperidol) repeated-measures analyses of variance (RM-ANOVA) was applied to analyze CO levels. A group $\times$ medication $\times$ picture (smoking-related pictures $v s$ neutral pictures) RM-ANOVA was performed to investigate reaction times and accuracy during line counting. QSU craving scores in smokers were analyzed with medication as a single within-subject factor. To investigate the effect of task performance on craving levels, a medication $\times$ time (before $v s$ after task performance) RM-ANOVA was performed for craving VAS scores.

\section{Image Acquisition}

Imaging data were acquired on a 3T GE Healthcare (The Discovery MRI 750 3.0T, Milwaukee, WI) scanner. Blood oxygen level-dependent sensitive functional $\mathrm{T} 2{ }^{\star}$-weighted images were acquired in 44 axial slices covering the entire supratentorial brain with a repetition time (TR) of $2500 \mathrm{~ms}$, echo time (TE) of $30 \mathrm{~ms}$, field of view (FOV) of $240 \mathrm{~mm}$, and isotropic voxel size of $2.5 \mathrm{~mm}^{3}$. A structural three-dimensional (3D) inversion recovery fast spoiled gradient echo T1-weighted image was acquired in 164 contiguous axial slices with TR of $7.9 \mathrm{~ms}$, TE of $3.1 \mathrm{~ms}$, FOV of $240 \mathrm{~mm}$, and isotropic voxel size of $1 \mathrm{~mm}^{3}$ for anatomical reference.

\section{Image Processing}

Imaging data were analyzed using SPM8 (Statistical Parametric Mapping; Wellcome Trust Centre for Neuroimaging, London, UK). Preprocessing of the functional data included realignment and unwarping of functional images. The anatomical scan was co-registered to the mean $2^{*}$ weighted image and subsequently segmented into grey and white matter. Segmentation parameters were used for normalization using the SPM T1 MNI template. Functional scans were spatially smoothed using a full-width at halfmaximum Gaussian kernel of $8 \mathrm{~mm}$. Correct trials for the four conditions (LCSP, LCNP, PNSP, and PNNP) were modeled in the context of the general linear model for both medication conditions, using delta functions convolved with a canonical hemodynamic response function. Incorrect trials were modeled separately as regressors of non-interest. The contrast reflecting brain activation associated with attentional bias (LCSP minus LCNP) was calculated for each individual for both medication conditions. Subsequently, a random effects RM-ANOVA with group as between-subject factor and medication as within-subject factor was performed to investigate group $\times$ medication interactions. Between group and between medication $t$-tests were performed (ie, differences between groups for placebo and haloperidol separately and medication effects in smokers and non-smokers separately), masked inclusively by voxels

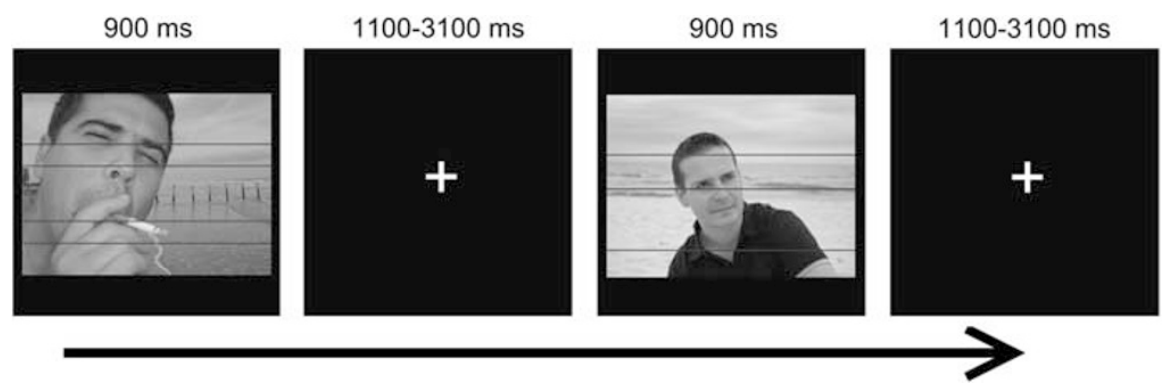

Figure I The attentional bias line-counting task. 


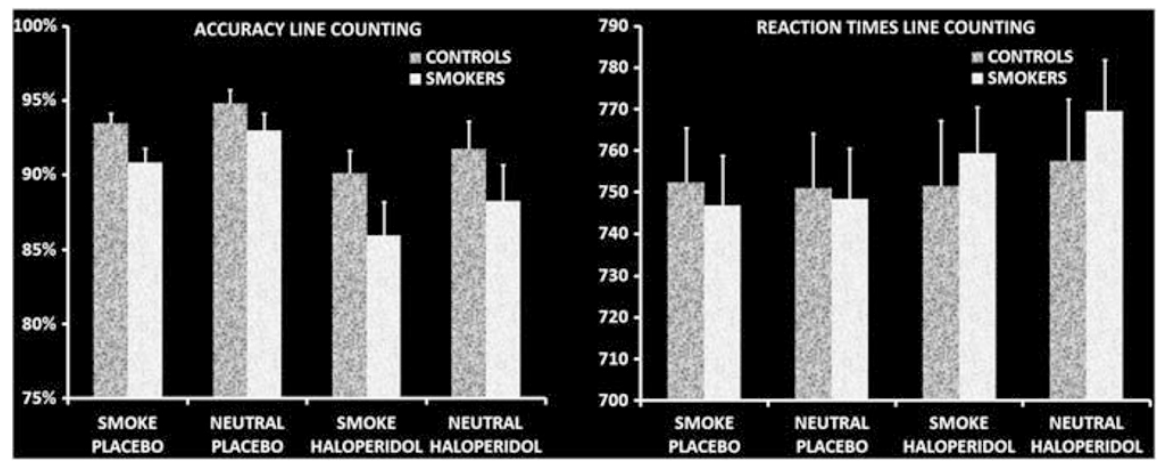

Figure 2 Behavioral measures for the attentional bias line-counting task. Repeated-measures analyses of variance for accuracy rates and reaction times during line counting showed that both smokers and non-smoking controls were less accurate for smoking-related pictures and showed shorter reaction times for smoking-related pictures. Both groups were less accurate after haloperidol.

showing a group $\times$ medication interaction in the RMANOVA $(p<0.01$ uncorrected), thus ensuring that group differences and medication effects met the requirement of a group $\times$ medication interaction. Furthermore, we report results for the between group two sample $t$-test for placebo without masking for the interaction effect, with the aim to replicate findings from our previous study (Luijten et al, 2011). Finally, cue-induced craving during task performance was calculated for each smoker for placebo and haloperidol separately (craving VAS score after task performance minus craving VAS score before task performance) and was correlated with brain activation associated with attentional bias in each medication condition separately. Given findings of previous studies the ACC, superior parietal lobe (SPL), superior temporal gyrus, dorsolateral prefrontal gyrus (DLPFC), inferior frontal gyrus, amygdala, insula, and nucleus accumbens (NACC) were selected as $a$ priori regions of interest. ROIs were defined using the automatic anatomical labeling (AAL) atlas (Tzourio-Mazoyer et al, 2002). As the NACC is not included in the AAL atlas, a $10 \mathrm{~mm}$ sphere with MNI coordinates $\pm 1012-2$ was created as a ROI for the NACC (Knutson et al, 2008). Results were thresholded at $p<0.05$, family wise error (FWE) corrected for multiple comparisons across the search volume (small volume correction: Friston et al, 1996; Worsley et al, 1996). In order to do so, analyses were first thresholded at $p<0.001$ uncorrected with 20 contingently activated voxels $\left(160 \mathrm{~mm}^{3}\right)$, and then corrected using a small volume correction ( $p<0.05$ FWE corrected) in which the search volume was defined by the AAL template corresponding to the a priori defined ROI.

\section{RESULTS}

\section{Breath CO Levels and Questionnaire Data}

Smokers had a higher breath $\mathrm{CO}$ concentration $\left(M_{\text {haloperidol }}=6.20, \mathrm{SD}=3.39, M_{\text {placebo }}=6.72, \mathrm{SD}=3.50\right)$ than non-smokers $\left(M_{\text {haloperidol }}=1.42, \mathrm{SD}=0.78, M_{\text {placebo }}=1.67\right.$, $\mathrm{SD}=0.64), \mathrm{F}(1,47)=55.15, p<0.001$. CO levels did not differ between medication conditions, $\mathrm{F}(1,47)=1,91$, NS. Haloperidol did not influence QSU $\left(M_{\text {haloperidol }}=38.03\right.$, $\left.\mathrm{SD}=11.80, M_{\text {placebo }}=39.71, \mathrm{SD}=11.48\right)$ and VAS craving scores. However, VAS craving scores increased after task performance confirming the presence of cue-evoked craving,
$\mathrm{F}(1,24)=21.36, p<0.001,\left(M_{\text {haloperidol/before }}=58.96, \mathrm{SD}=21.49\right.$, $M_{\text {haloperidol/after }}=67.00, \quad \mathrm{SD}=17.84, \quad M_{\text {placebo/before }}=62.60$, $\left.\mathrm{SD}=23.21, M_{\text {placebo/after }}=68.64, \mathrm{SD}=23.38\right)$.

\section{Behavioral Performance}

Accuracy scores and reaction times are displayed in Figure 2. Repeated-measures analysis for performance accuracy did not show a main effect of group $\mathrm{F}(1,47)=2.96$, NS. A main effect of medication showed that haloperidol decreased task performance relative to placebo, $\mathrm{F}(1,47)=10.36, p<0.01$. Furthermore, a main effect of picture was found for accuracy $(\mathrm{F}(1,47)=11.10$, $p<0.01$ ), with both groups performing less accurately for line counting in smoking-related pictures than in neutral pictures. Regarding reaction times, no main effect of group or medication was found, both F value's $<3.71$, NS. A main effect of picture was found, $\mathrm{F}(1,37)=4.14, \quad p<0.05$, indicating that reaction times to smoking-related pictures were faster. No interaction effects were found, all $\mathrm{F}$ value's $<2.87$, NS. The combination of reduced accuracy and faster reaction times to smoking-related pictures suggests that there may be an impulsive response style to smoking-related pictures in both groups.

\section{Imaging Results}

After placebo, smokers showed attentional bias-related brain activation (ie, more activation than controls on the LCSP minus LCNP contrast) in the dorsal zone of the ACC (dACC), the left SPL and the right DLPFC (r-DLPFC). After masking for the group $\times$ medication interaction, group differences remained present in the dACC and the r-DLPFC. No differences between groups were found in attentional bias-related brain activation after haloperidol administration. See Figure 3 and Table 1 for details. These findings suggest that the dACC, r-DLPFC left SPL are involved in attentional bias in smokers. Group $\times$ medication interactions and the lack of group differences after haloperidol suggest that brain activation associated with attentional bias in smokers is normalized when dopamine levels are reduced by haloperidol. Paired $t$-tests, however, did not reveal significant medication effects in either smokers or nonsmokers at the FWE-corrected level. We therefore extracted parameter estimates in those regions showing a whole brain 


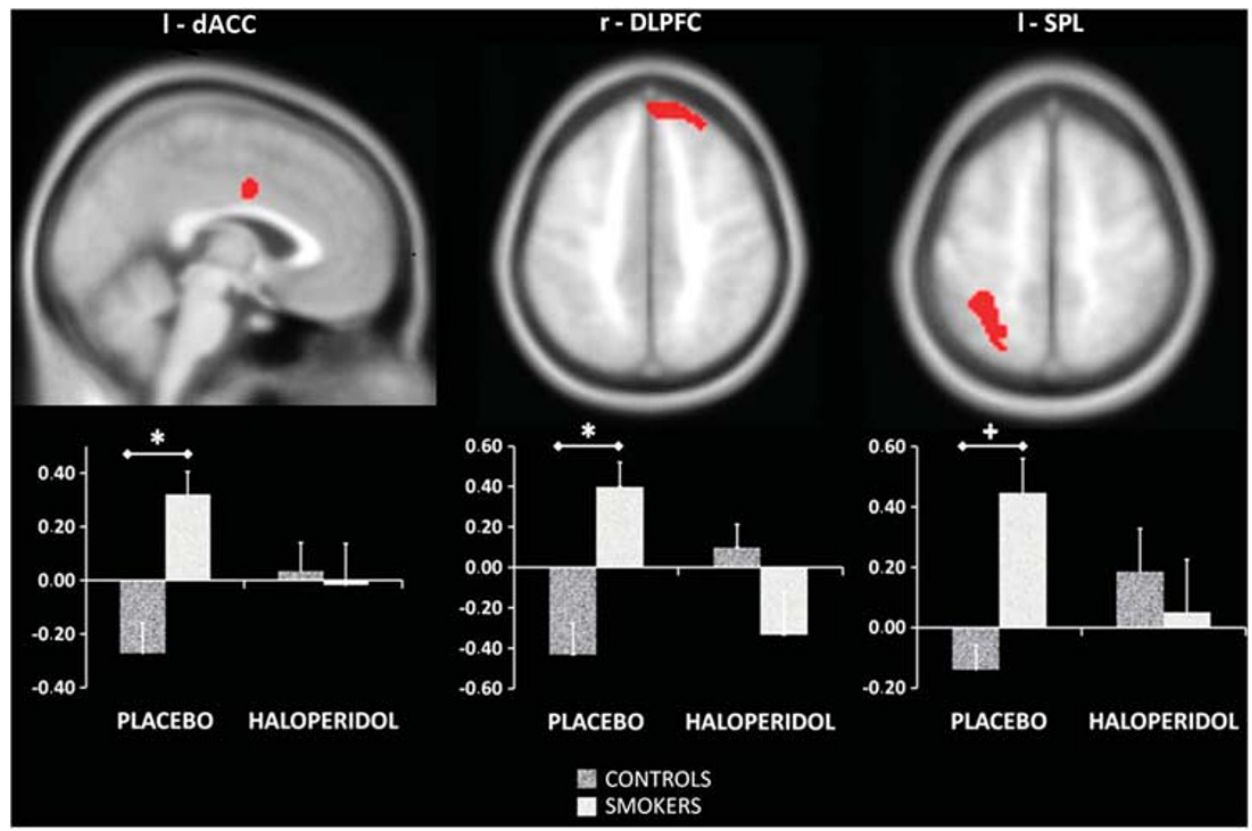

Figure 3 Group differences for brain activation associated with attentional bias. * $p<0.05$ FWE small volume corrected both with and without masking for the group $\times$ medication interaction, ${ }^{+} p<0.05$ FWE small volume corrected only without masking for the group $\times$ medication interaction. The values on the $Y$ axis represent contrast values for LCSP minus LCNP, consequently positive values on the $Y$ axis indicate more brain activation for line counting when smoking-related pictures are presented on the background relative to when neutral pictures are presented on the background. FWE, family wise error; LCNP, line-counting neutral picture; LCSP, line-counting smoke picture; I, left; r, right; dACC, dorsal anterior cingulate gyrus; DLPFC, dorsolateral prefrontal cortex; SPL, superior parietal lobe.

group $\times$ medication interaction and significant group differences (dACC and r-DLPFC). Subsequently, paired sample $t$-tests in SPSS were performed for both groups separately. Results showed a significant reduction in brain activation in smokers for the r-DLPFC, $t(24)=3.07, p<0.01$, and a trend for the dACC, $t(24)=1.99, p<0.058$. Brain activation in the r-DLPFC was significantly increased by haloperidol for nonsmokers, $t(23)=2.67, p<0.05$.

Brain activation associated with attentional bias in smokers was neither positively nor negatively correlated with cueevoked craving during task performance in any of our $a$ priori defined regions of interest. See Supplementary Tables 7 and 8 for (nonsignificant) correlation coefficients between craving and brain and behavioral indices of attentional bias.

\section{DISCUSSION}

The main purpose of this study was to investigate whether brain activation associated with attentional bias in smokers could be modulated by a dopamine antagonist. The current results provide support for the proposed role of dopamine in attentional bias. In line with our hypotheses, smokers showed increased activation associated with attentional bias in the dACC, right DLPFC, and left SPL after placebo, whereas this activation was normalized when dopamine levels were reduced following administration of a dopamine antagonist. That is, no differences in brain activation between smokers and non-smokers were found after haloperidol intake. These results are in line with a previous study showing that the dopamine antagonist amisulpride normalized cue-induced brain activation in alcohol-dependent patients (Hermann et al, 2006).

The current findings replicate and extend findings of our previous study using the same pictorial attentional bias task (Luijten et al, 2011). Again, our results implicate a role for the dACC in attentional bias in smokers. The dACC is known to be involved in multiple cognitive processes (Shackman et al, 2011) such as salience detection (Seeley et al, 2007), behavioral monitoring, and top-down control of attention (Bush et al, 2000; Weissman et al, 2005). The dopaminergic signal in the striatum evoked by conditioned substance cues (Volkow et al, 2006; Wong et al, 2006; Zijlstra et al, 2008) may modulate dACC activation via connections between the dACC and the ventral striatum (Kunishio and Haber, 1994) such that the salience of these cues is detected. Meanwhile, the dACC may signal conflict of attentional resources, because attention is automatically allocated to the substance cues and withdrawn from ongoing behavior. Given the multi-functionality of the dACC (Shackman et al, 2011), we suggest that the dACC is involved in salience detection of conditioned substance cues and subsequent allocation of additional cognitive resources. To increase cognitive control for the continuation of ongoing behavior during smoking-cue exposure, the ACC may cooperate with the DLPFC, a region that was also found to be associated with attentional bias in smokers. For example, it has been shown that coactivation of the dACC and DLPFC contributes to the implementation of adjustments in activation of future behavior (Kerns et al, 2004). In this study, we showed that when dopamine transmission in response to conditioned smoking cues is reduced by a dopamine antagonist, the activation in the dACC and 
Table I Group Differences for Brain Activation Associated with Attentional Bias

\begin{tabular}{|c|c|c|c|c|}
\hline \multicolumn{3}{|c|}{ MNI coordinates } & \multirow[t]{2}{*}{ Z-value $^{a}$} & \multirow[t]{2}{*}{$\mathrm{mm}^{3}$} \\
\hline$x$ & $\mathbf{Y}$ & $\mathbf{Z}$ & & \\
\hline
\end{tabular}

Placebo

Smokers $>$ controls

Left dACC $\quad-2$

Right DLPFC

Left $\mathrm{SPL}^{\mathrm{C}}$

$-2$

-2
44
-46

36
46
56

4.00

4.03

3.85

$360^{\mathrm{b}}$

$-34$

$-46$

56

456

616

Smokers $<$ controls

Haloperidol

Smokers $>$ controls

Smokers $<$ controls

Abbreviations: dACC: dorsal anterior cingulate cortex; DLPFC, dorsolateral prefrontal gyrus; SPL, superior parietal lobe.

${ }^{a} p<0.05$ FWE small volume corrected.

${ }^{\mathrm{b}}$ After masking with the group $\times$ medication interaction the size of this cluster reduced to $176 \mathrm{~mm}^{3}$.

${ }^{c}$ After masking with the group $\times$ medication interaction this cluster no longer met the requirements to correct for multiple comparisons.

DLPFC associated with attentional bias in smokers is reduced accordingly. For future studies, it would be interesting to examine whether individual differences in dopaminergic activation in smokers are associated with differences in attentional bias-related brain activation. Although the dopaminergic theory for attentional bias does not involve an inverted U-shape aspect as yet, it may be that the association between dopamine and the attentional control aspects of attentional bias, follows a similar inverted U-shape curve as previously described in the domain of cognitive control (Cools and D'Esposito, 2011). In studies addressing this hypothesis, a group of smokers with a broad range of attentional bias scores should be included to sample all parts of the U-shaped curve. Also, dopamine levels would preferably be measured with PET in order to obtain a more direct estimation of dopamine levels.

The findings in this study provide a proof of principles for the role of dopamine in attentional bias-related brain activation and may guide the development of new pharmacotherapies for smoking addiction. However, some findings in this study suggest that the association between dopamine and controlling substance-related behavioral may be rather complex. First, it was found that haloperidol reduced overall performance accuracy and activation in the medial prefrontal and bilateral DLPFC during line counting (see Supplementary Table 5) suggesting that dopamine antagonists may reduce overall cognitive control. Given that reduced cognitive control is also associated with problems controlling substance use (Feil et al, 2010; Goldstein and Volkow, 2011), a reduction in cognitive control may constitute an unfavorable effect. Second, the single dose of haloperidol was not able to reduce subjective craving in smokers, which is in line with previous studies failing to show reduced craving after a short term reduction in dopamine levels (Ersche et al, 2010; Franken et al, 2004; Hitsman et al, 2008; Munafo et al, 2007). Various explanations exist for the discrepancy in findings between attentional bias-related brain activation and subjective craving. First, we could not replicate the association between attentional bias-related brain activation and subjective craving as shown in our previous study (Luijten et al, 2011). This inconsistency remains currently unresolved, as previous imaging studies investigating attentional bias in addicted individuals have not examined this association (Ersche et al, 2010; Hester and Garavan, 2009; Janes et al, 2010a, b; Nestor et al, 2011). The discrepancy in effects of the dopaminergic manipulation as well as the lack of a consistent association between attentional bias-related brain activation and craving may arise as a consequence of differences between phasic and tonic dopamine neurotransmission. Phasic dopamine transmission has been suggested to be involved in attentional bias, whereas tonic dopamine levels may mediate symptoms associated with withdrawal such as subjective craving (Hitsman et al, 2008). Furthermore, it has been proposed that brain activation is a rather sensitive measure to detect abnormalities in addicted individuals regarding cue reactivity compared to other subjective measures such as craving (Goldstein and Volkow, 2011). The latter is also in line with behavioral data in this study that could not differentiate between smokers and non-smokers. In fact, behavioral data indicated that smoking-related pictures disrupt ongoing behavior in both groups indicating that brain activation differences between groups after placebo should be interpreted as increased neural effort in smokers to reach similar performance. Enhanced activation in non-smokers for picture naming of smoking-related vs neutral pictures (see Supplementary Table 4) further support that non-smokers may react stronger to smoking cues. Although interference for smoking cues in non-smokers is not typically found, a previous study that also showed this interference effect in non-smokers suggested that it may be due to non-addiction reasons such as negative valence (Stippekohl et al, 2012). Another unexpected finding in non-smokers is that brain activation associated with attentional bias after haloperidol was significantly increased. Activation patterns (see Figure 3) suggest that non-smokers are characterized by reduced activation for line counting in smoking-related pictures relative to neutral pictures after placebo, an effect that disappeared after haloperidol administration. Although highly speculative, we suggest that the increase in brain activation in the DLPFC for smoking-related pictures reflects an attempt to prevent a further decrement in performance levels as performance is lowest after haloperidol and for smoking-related pictures in general.

A final important consideration regarding this study is that smokers did not smoke for $4 \mathrm{~h}$ before testing as this could have interfered with medication effects. Given this study design, we cannot completely rule out withdrawal effects on our results. We could demonstrate, however, that withdrawal was not influenced by medication type and was not associated with individual differences in task performance 
(see Supplementary materials). In our previous study showing similar attentional bias-related brain activation (Luijten et al, 2011), smokers were abstinent for $3 \mathrm{~h}$, whereas smokers smoked did not abstain in other studies investigating attentional bias-related brain activation in smokers (Janes et al, 2010a, b; Nestor et al, 2011; Stippekohl et al, 2012). Generally, it is assumed that attentional bias is augmented after longer periods of abstinence (Waters and Feyerabend, 2000), therefore the $4 \mathrm{~h}$ abstinence period is important to consider when interpreting the current results.

To conclude, it was shown that administration of a dopamine antagonist normalized activation associated with attentional bias in the AACC and DLPFC in smokers. This finding supports theoretical accounts of the role of dopamine in attentional bias, and may have implications for the development of new pharmacotherapies for smoking addiction. However, our finding that haloperidol reduced overall task performance and associated brain activation indicates that it should be a future research agenda to investigate whether an optimal balance of dopamine in different brain regions in smokers can be achieved.

\section{ACKNOWLEDGEMENTS}

We thank Esther Spittel and Michelle Snelleman for their assistance with data collection, participant recruitment, and data processing.

\section{DISCLOSURE}

The authors declare that this work was funded by a grant of the Netherlands Organization for Scientific Research (NWO; VIDI grant for IHA Franken, number 016.08.322). The funding organization had no role in study design, data collection and analysis, decision to publish, or preparation of the manuscript. The authors declare no conflict of interest.

\section{REFERENCES}

Attwood AS, O'Sullivan H, Leonards U, Mackintosh B, Munafo MR (2008). Attentional bias training and cue reactivity in cigarette smokers. Addiction 103: 1875-1882.

Bush G, Luu P, Posner MI (2000). Cognitive and emotional influences in anterior cingulate cortex. Trends Cogn Sci 4: 215-222.

Carpenter KM, Schreiber E, Church S, McDowell D (2006). Drug Stroop performance: relationships with primary substance of use and treatment outcome in a drug-dependent outpatient sample. Addict Behav 31: 174-181.

Cools R, D’Esposito M (2011). Inverted-U-shaped dopamine actions on human working memory and cognitive control. Biol Psychiatry 69: e113-e125.

Cox WM, Hogan LM, Kristian MR, Race JH (2002). Alcohol attentional bias as a predictor of alcohol abusers' treatment outcome. Drug Alcohol Depend 68: 237-243.

Ersche KD, Bullmore ET, Craig KJ, Shabbir SS, Abbott S, Muller U et al (2010). Influence of compulsivity of drug abuse on dopaminergic modulation of attentional bias in stimulant dependence. Arch Gen Psychiatry 67: 632-644.

Fadardi JS, Cox WM (2009). Reversing the sequence: reducing alcohol consumption by overcoming alcohol attentional bias. Drug Alcohol Depend 101: 137-145.

Feil J, Sheppard D, Fitzgerald PB, Yücel M, Lubman DI, Bradshaw JL (2010). Addiction, compulsive drug seeking, and the role of frontostriatal mechanisms in regulating inhibitory control. Neurosci Biobehav Rev 35: 248-275.

Field M, Cox WM (2008). Attentional bias in addictive behaviors: a review of its development, causes, and consequences. Drug Alcohol Depend 97: 1-20.

Field M, Duka T, Tyler E, Schoenmakers T (2009a). Attentional bias modification in tobacco smokers. Nicotine Tob Res 11: 812-822.

Field M, Munafo MR, Franken IH (2009b). A meta-analytic investigation of the relationship between attentional bias and subjective craving in substance abuse. Psychol Bull 135: 589-607.

Franken IH (2003). Drug craving and addiction: integrating psychological and neuropsychopharmacological approaches. Prog Neuropsychopharmacol Biol Psychiatry 27: 563-579.

Franken IH, Nijs I, Pepplinkhuizen L (2008). Effects of dopaminergic modulation on electrophysiological brain response to affective stimuli. Psychopharmacology (Berl) 195: 537-546.

Franken IH, Stam CJ, Hendriks VM, van den Brink W (2003). Neurophysiological evidence for abnormal cognitive processing of drug cues in heroin dependence. Psychopharmacology (Berl) 170: 205-212.

Franken IHA, Hendriks VM, Stam CJ, Van den Brink W (2004). A role for dopamine in the processing of drug cues in heroin dependent patients. Eur Neuropsychopharmacol 14: 503-508.

Friston KJ, Holmes A, Poline JB, Price CJ, Frith CD (1996). Detecting activations in PET and fMRI: levels of inference and power. Neuroimage 4: 223-235.

Goldstein RZ, Volkow ND (2011). Dysfunction of the prefrontal cortex in addiction: neuroimaging findings and clinical implications. Nat Rev Neurosci 12: 652-669.

Heatherton TF, Kozlowski LT, Frecker RC, Fagerstrom KO (1991). The Fagerstrom test for nicotine dependence: a revision of the fagerstrom tolerance questionnaire. $\mathrm{Br} J$ Addict 86: 1119-1127.

Hermann D, Smolka MN, Wrase J, Klein S, Nikitopoulos J, Georgi A et al (2006). Blockade of cue-induced brain activation of abstinent alcoholics by a single administration of amisulpride as measured with fMRI. Alcohol Clin Exp Res 30: 1349-1354.

Hester R, Garavan H (2009). Neural mechanisms underlying drugrelated cue distraction in active cocaine users. Pharmacol Biochem Behav 93: 270-277.

Hitsman B, MacKillop J, Lingford-Hughes A, Williams TM, Ahmad F, Adams S et al (2008). Effects of acute tyrosine/phenylalanine depletion on the selective processing of smoking-related cues and the relative value of cigarettes in smokers. Psychopharmacology (Berl) 196: 611-621.

Janes AC, Pizzagalli DA, Richardt S, deB Frederick B, Chuzi S, Pachas $\mathrm{G}$ et al (2010a). Brain reactivity to smoking cues prior to smoking cessation predicts ability to maintain tobacco abstinence. Biol Psychiatry 67: 722-729.

Janes AC, Pizzagalli DA, Richardt S, Frederick Bde B, Holmes AJ, Sousa $J$ et al (2010b). Neural substrates of attentional bias for smoking-related cues: an FMRI study. Neuropsychopharmacology 35: 2339-2345.

Kerns JG, Cohen JD, MacDonald AW, Cho RY, Stenger VA, Carter CS (2004). Anterior cingulate conflict monitoring and adjustments in control. Science 303: 1023-1026.

Knutson B, Wimmer GE, Rick S, Hollon NG, Prelec D, Loewenstein G (2008). Neural antecedents of the endowment effect. Neuron 58: $814-822$.

Kuhn S, Gallinat J (2011). Common biology of craving across legal and illegal drugs - a quantitative meta-analysis of cue-reactivity brain response. Eur J Neurosci 33: 1318-1326.

Kunishio K, Haber SN (1994). Primate cingulostriatal projection: limbic striatal versus sensorimotor striatal input. J Comp Neurol 350: $337-356$.

Luijten M, Veltman DJ, den Brink WV, Hester R, Field M, Smits M et al (2011). Neurobiological substrate of smoking-related attentional bias. Neuroimage 54: 2374-2381. 
Mahler SV, de Wit H (2005). Effects of haloperidol on reactions to smoking cues in humans. Behav Pharmacol 16: 123-126.

Marissen MA, Franken IH, Waters AJ, Blanken P, van den Brink W, Hendriks VM (2006). Attentional bias predicts heroin relapse following treatment. Addiction 101: 1306-1312.

McHugh RK, Murray HW, Hearon BA, Calkins AW, Otto MW (2010). Attentional bias and craving in smokers: the impact of a single attentional training session. Nicotine Tob Res 12: 1261-1264.

Munafo MR, Mannie ZN, Cowen PJ, Harmer CJ, McTavish SB (2007). Effects of acute tyrosine depletion on subjective craving and selective processing of smoking-related cues in abstinent cigarette smokers. J Psychopharmacol 21: 805-814.

Nestor L, McCabe E, Jones J, Clancy L, Garavan H (2011). Differences in 'bottom-up' and 'top-down' neural activity in current and former cigarette smokers: Evidence for neural substrates which may promote nicotine abstinence through increased cognitive control. Neuroimage 56: 2258-2275.

Nordstrom AL, Farde L, Halldin C (1992). Time course of D2dopamine receptor occupancy examined by PET after single oral doses of haloperidol. Psychopharmacology (Berl) 106: 433-438.

Phillips PE, Stuber GD, Heien ML, Wightman RM, Carelli RM (2003). Subsecond dopamine release promotes cocaine seeking. Nature 422: 614-618.

Robbins SJ, Ehrman RN (2004). The role of attentional bias in substance abuse. Behav Cogn Neurosci Rev 3: 243-260.

Robinson TE, Berridge KC (2003). Addiction. Annu Rev Psychol 54: 25-53.

Robinson TE, Berridge KC (2008). Review. The incentive sensitization theory of addiction: some current issues. Philos Trans R Soc Lond B Biol Sci 363: 3137-3146.

Schoenmakers TM, de Bruin M, Lux IFM, Goertz AG, Van Kerkhof DHAT, Wiers RW (2010). Clinical effectiveness of attentional bias modification training in abstinent alcoholic patients. Drug Alcohol Depend 109: 30-36.

Seeley WW, Menon V, Schatzberg AF, Keller J, Glover GH, Kenna H et al (2007). Dissociable intrinsic connectivity networks for salience processing and executive control. J Neurosci 27: 2349-2356.

Shackman AJ, Salomons TV, Slagter HA, Fox AS, Winter JJ, Davidson RJ (2011). The integration of negative affect, pain and cognitive control in the cingulate cortex. Nat Rev Neurosci 12: 154-167.

Stippekohl B, Walter B, Winkler MH, Mucha RF, Pauli P, Vaitl D et al (2012). An early attentional bias to BEGIN-stimuli of the smoking ritual is accompanied with mesocorticolimbic deactivations in smokers. Psychopharmacology (Berl); e-pub ahead of print 3 April 2012.

Tzourio-Mazoyer N, Landeau B, Papathanassiou D, Crivello F, Etard O, Delcroix N et al (2002). Automated anatomical labeling of activations in SPM using a macroscopic anatomical parcellation of the MNI MRI single-subject brain. Neuroimage 15: 273-289.

Vink JM, Willemsen G, Beem AL, Boomsma DI (2005). The Fagerström test for nicotine dependence in a Dutch sample of daily smokers and ex-smokers. Addict Behav 30: 575-579.

Volkow ND, Wang GJ, Telang F, Fowler JS, Logan J, Childress AR et al (2006). Cocaine cues and dopamine in dorsal striatum: mechanism of craving in cocaine addiction. J Neurosci 26: 6583-6588.

Vollstadt-Klein S, Loeber S, Richter A, Kirsch M, Bach P, von der Goltz C et al (2012). Validating incentive salience with functional magnetic resonance imaging: association between mesolimbic cue reactivity and attentional bias in alcohol-dependent patients. Addict Biol 17: 807-816.

Waters AJ, Feyerabend C (2000). Determinants and effects of attentional bias in smokers. Psychol Addict Behav 14: 111-120.

Waters AJ, Marhe R, Franken IH (2012). Attentional bias to drug cues is elevated before and during temptations to use heroin and cocaine. Psychopharmacology (Berl) 219: 909-921.

Waters AJ, Shiffman S, Sayette MA, Paty JA, Gwaltney CJ, Balabanis MH (2003). Attentional bias predicts outcome in smoking cessation. Health Psychol 22: 378-387.

Weissman DH, Gopalakrishnan A, Hazlett CJ, Woldorff MG (2005). Dorsal anterior cingulate cortex resolves conflict from distracting stimuli by boosting attention toward relevant events. Cereb Cortex 15: 229-237.

Wong DF, Kuwabara H, Schretlen DJ, Bonson KR, Zhou Y, Nandi A et al (2006). Increased occupancy of dopamine receptors in human striatum during cue-elicited cocaine craving. Neuropsychopharmacology 31: 2716-2727.

Worsley KJ, Marrett S, Neelin P, Vandal AC, Friston KJ, Evans AC (1996). A unified statistical approach for determining significant signals in images of cerebral activation. Hum Brain Mapp 4: $58-73$.

Zijlstra F, Booij J, van den Brink W, Franken IH (2008). Striatal dopamine D2 receptor binding and dopamine release during cue-elicited craving in recently abstinent opiate-dependent males. Eur Neuropsychopharmacol 18: 262-270. 